\title{
Clinical Outcomes With Abemaciclib After Prior CDK4/6 Inhibitor Progression in Breast Cancer: A Multicenter Experience
}

\author{
Seth A. Wander, MD, PhD 1,2,*; Hyo S. Han, MD 3,*; Mark L. Zangardi, PharmD; Andrzej Niemierko, PhD 1,2; \\ Veronica Mariotti, MD; Leslie S.L. Kim, BS ${ }^{4}$; Jing Xi, MD'; Apurva Pandey, MD ${ }^{6}$; Siobhan Dunne, BA ${ }^{4}$; \\ Azadeh Nasrazadani, MD, PhD ${ }^{6}$; Avinash Kambadakone, MD ${ }^{1,2}$; Casey Stein, BS ${ }^{1}$; Maxwell R. Lloyd, BS ${ }^{1}$; Megan Yuen, BS ${ }^{1,2}$; \\ Laura M. Spring, MD ${ }^{1,2}$; Dejan Juric, MD ${ }^{1,2}$; Irene Kuter, MD ${ }^{1,2}$; loannis Sanidas, PhD ${ }^{1,2}$; Beverly Moy, MD, MPH ${ }^{1,2}$; \\ Therese Mulvey, MD ${ }^{1,2}$; Neelima Vidula, MD ${ }^{1,2}$; Nicholas J. Dyson, $\mathrm{PhD}^{1,2}$; Leif W. Ellisen, MD, $\mathrm{PhD}^{1,2}$; \\ Steven Isakoff, MD, PhD ${ }^{1,2}$; Nikhil Wagle, MD, PhD 2,7,8; Adam Brufsky, MD, PhD ${ }^{6}$; Kevin Kalinsky, MD ; \\ Cynthia X. Ma, MD, $\mathrm{PhD}^{5}$; Joyce O'Shaughnessy, $\mathrm{MD}^{4}$; and Aditya Bardia, MD, MPH ${ }^{1,2}$
}

\begin{abstract}
Background: Inhibitors of cyclin-dependent kinases 4 and 6 (CDK4/ 6i) are widely used as first-line therapy for hormone receptor-positive metastatic breast cancer (HR+ MBC). Although abemaciclib monotherapy is also FDA-approved for treatment of disease progression on endocrine therapy, there is limited insight into the clinical activity of abemaciclib after progression on prior CDK4/6i. Patients and Methods: We identified patients with HR+ MBC from 6 cancer centers in the United States who received abemaciclib after disease progression on prior CDK4/6i, and abstracted clinical features, outcomes, toxicity, and predictive biomarkers. Results: In the multicenter cohort, abemaciclib was well tolerated after a prior course of CDK4/6i (palbociclib)-based therapy; a minority of patients discontinued abemaciclib because of toxicity without progression (9.2\%). After progression on palbociclib, most patients $(71.3 \%)$ received nonsequential therapy with abemaciclib (with $\geq 1$ intervening non-CDK4/6i regimens), with most receiving abemaciclib with an antiestrogen agent (fulvestrant, 47.1\%; aromatase inhibitor, $27.6 \%$ ), and the remainder receiving abemaciclib monotherapy (19.5\%). Median progression-free survival for abemaciclib in this population was 5.3 months and median overall survival was 17.2 months, notably similar to results obtained in the MONARCH-1 study of abemaciclib monotherapy in heavily pretreated HR+/HER2-negative CDK4/6i-naïve patients. A total of $36.8 \%$ of patients received abemaciclib for $\geq 6$ months. There was no relationship between the duration of clinical benefit while on palbociclib and the subsequent duration of treatment with abemaciclib. RB1, ERBB2, and CCNE1 alterations were noted among patients with rapid progression on abemaciclib. Conclusions: A subset of patients with HR+ MBC continue to derive clinical benefit from abemaciclib after progression on prior palbociclib. These results highlight the need for future studies to confirm molecular predictors of cross-resistance to CDK4/6i therapy and to better characterize the utility of abemaciclib after disease progression on prior CDK4/6i.
\end{abstract}

J Natl Compr Canc Netw, doi: 10.6004/jnccn.2020.7662 Published online March 24, 2021

See JNCCN.org for supplemental online content.

\section{Background}

The treatment landscape for patients with hormone receptor-positive (HR+), HER2-negative (HER2-) metastatic breast cancer (MBC) has evolved with the advent of targeted agents that demonstrate synergism with antiestrogens. Inhibitors of cyclin-dependent kinases 4 and 6 (CDK4/6i) have emerged as standard of care, in combination with an antiestrogen, in either the first-line setting, or after progression on endocrine therapy. ${ }^{1}$

Multiple large, randomized studies have demonstrated improvements in progression-free survival (PFS) for three CDK 4/6i agents (palbociclib, ribociclib, abemaciclib) in combination with an antiestrogen in $\mathrm{HR}+1$ HER- MBC..$^{2-6}$ Updated analyses have also demonstrated significant improvement in overall survival (OS) with the combination of endocrine therapy and abemaciclib or ribociclib. ${ }^{7-9}$ A nonsignificant trend toward improved OS was also appreciated in updated analyses exploring combinations with palbociclib. ${ }^{10,11}$ These agents are well tolerated and common toxicities include leukopenia/neutropenia, fatigue, diarrhea (for abemaciclib), and rare instances of QTc prolongation (for ribociclib). ${ }^{1}$ Although palbociclib and ribociclib have similar pharmacodynamic and pharmacokinetic properties (with overlapping toxicity profiles), there has been

\footnotetext{
${ }^{1}$ Massachusetts General Hospital Cancer Center, and ${ }^{2}$ Harvard Medical School, Boston, Massachusetts; ${ }^{3}$ Moffitt Cancer Center, Tampa, Florida; ${ }^{4}$ Baylor University Medical Center, Texas Oncology, US Oncology, Dallas, Texas;

${ }^{5}$ Washington University, St. Louis, Missouri; ${ }^{6}$ University of Pittsburgh, Pittsburgh, Pennsylvania; ${ }^{7}$ Dana-Farber Cancer Institute, and ${ }^{8}$ Broad Institute of MIT and Harvard, Boston, Massachusetts; and ${ }^{9}$ Columbia University Irving Medical Center, New York, New York.
}

*These authors contributed equally to this work. 
increasing interest in the application of abemaciclib given its unique dosing schedule (continuous vs intermittent), increased selectivity for CDK4 over CDK6, ability to cross the blood-brain barrier, and single-agent activity. ${ }^{12,13}$ Abemaciclib, in contrast with palbociclib and ribociclib, also appears to inhibit CDK2 and CDK1 via proteomic and transcriptional analyses. ${ }^{14}$

Abemaciclib has demonstrated efficacy as a single agent in heavily pretreated patients with HR+/HER2MBC based on the phase II MONARCH-1 trial, a singlearm study exploring the clinical activity of abemaciclib monotherapy (200 mg, twice daily) in patients with endocrine therapy-refractory HR+/HER2- MBC. ${ }^{15}$ Median PFS was 6.0 months (95\% CI, 4.2-7.5 months) in this trial, the results of which led to approval of abemaciclib as monotherapy for CDK4/6i-naïve, hormone therapy-refractory patients. However, none of the patients in the MONARCH-1 trial had received a prior CDK4/6i, and the efficacy of abemaciclib post-CDK4/6i is unclear.

Despite widespread clinical experience with these agents, we have limited insight into the role of continued CDK4/ 6 blockade in patients who have received a prior CDK4/6i. Emerging data suggest that clinical resistance may be mediated by inactivation of $R B 1$; overexpression of $C D K 6, C C N E 1 / 2$, and aurora kinase A; and mutational activation of FGFR, ERBB2, AKT1, and $R A S$ family oncogenes. ${ }^{16-28}$ However, the clinical utility of these putative biomarkers for routine medical decision-making has not been established. Despite the lack of data, some clinicians administer a second course of CDK4/6 blockade after progression in the first- or second-line HR+/HER2- metastatic setting, similar to the longstanding practice of continuing HER2-directed therapy after progression on trastuzumab. In particular, there is interest in the potential activity of abemaciclib after disease progression on palbociclib- or ribociclib-based treatment given its unique pharmacokinetic and pharmacodynamic properties, and many clinicians use abemaciclib in this setting without available retrospective or prospective data to support this approach. Establishing better insight into the potential utility of ongoing CDK4/6 blockade after initial progression, particularly for abemaciclib, represents a critical unmet need in the current MBC treatment landscape.

We sought to address this question by identifying patients with HR+/HER2- MBC who experienced progression on palbociclib or ribociclib and subsequently received abemaciclib-based therapy. We report on the treatment patterns of continued CDK4/6i therapy across 6 institutions, the clinical outcome data, the toxicity experience with a second course of $\mathrm{CDK} 4 / 6 \mathrm{i}$, and emerging insights into the genomic predictors of resistance to abemaciclib in this patient population.

\section{Patients and Methods}

\section{Patient Selection}

Within each participating cancer center, patients with HR+/HER2 - MBC who had received abemaciclib at any time through May 1, 2019 were identified. Within this cohort, patients were identified who had also received a prior course of palbociclib or ribociclib in the metastatic setting. Inclusion details are outlined in supplemental eAppendix 1 (available with this article at JNCCN.org).

Medical records of eligible patients were analyzed for relevant clinical metrics, including age at initial cancer diagnosis, metastatic diagnosis, and death or most recent clinic visit date (if applicable). All medical records were deidentified prior to data analysis and were collected according to the requirements of each site's Institutional Review Board. Informed consent for data collection was obtained in accordance with the Declaration of Helsinki.

For each patient, the highest grade of the specific event of interest (via the CTCAE version 4.03) was noted if multiple episodes of an adverse effect occurred during the treatment course. Subjective adverse effects, including diarrhea, fatigue, and nausea, were not graded as part of this retrospective chart review.

\section{Statistical Analysis}

OS and PFS were estimated using the Kaplan-Meier method, and survival curves were compared using logrank test. The association of risk factors with outcome was analyzed using Cox proportional hazards method. All analyses were performed using STATA, version 16 (StataCorp LLC).

\section{Results}

We identified 87 patients with $\mathrm{HR}+\mathrm{MBC}$ from 6 medical centers in the United States who received abemaciclib after prior progression on a palbociclib- or ribociclibcontaining regimen in the metastatic setting.

Median age at metastatic diagnosis was 51.8 years (Table 1). All 87 patients received palbociclib as their initial line of CDK4/6i; none of the patients who met the eligibility criteria had received ribociclib.

\section{Abemaciclib Use After Prior CDK4/6i Therapy}

Given the lack of guidance from prospective randomized trials, practice patterns differed among physicians. Most patients received palbociclib in combination with an aromatase inhibitor (AI; $\mathrm{n}=55 ; 63.2 \%$ ), a subgroup received palbociclib + fulvestrant $(n=25 ; 28.7 \%)$, and none received palbociclib as a single agent. 


\section{Table 1. Clinical Parameters}

\begin{tabular}{|c|c|}
\hline Parameter & n (\%) \\
\hline \multicolumn{2}{|l|}{ Median age (range), y } \\
\hline Original diagnosis & $48.6(26.5-72.3)$ \\
\hline Metastatic diagnosis & $51.8(29.5-74.1)$ \\
\hline \multicolumn{2}{|l|}{ Sex } \\
\hline Female & $86(98.9)$ \\
\hline Male & $1(1.1)$ \\
\hline \multicolumn{2}{|c|}{ Median number of prior lines, metastatic setting (range) } \\
\hline Endocrine & $3(1-7)$ \\
\hline Nonendocrine & $2(0-12)$ \\
\hline Total & $5(1-15)$ \\
\hline \multicolumn{2}{|l|}{ CDK4/6i sequence } \\
\hline Nonsequential & $62(71.3)$ \\
\hline Sequential & $25(28.7)$ \\
\hline \multicolumn{2}{|c|}{ Abemaciclib status at data cutoff (May 1, 2019) } \\
\hline Receiving treatment & $21(24.1)$ \\
\hline Discontinued, progression & $58(66.7)$ \\
\hline Discontinued, toxicity & $8(9.2)$ \\
\hline \multicolumn{2}{|l|}{ Palbociclib treatment course } \\
\hline \multicolumn{2}{|l|}{ Endocrine partner } \\
\hline Aromatase inhibitor & $55(63.2)$ \\
\hline Fulvestrant & $25(28.7)$ \\
\hline Tamoxifen & $0(0.0)$ \\
\hline Multiple & $7(8.1)$ \\
\hline NA (monotherapy) & $0(0.0)$ \\
\hline \multicolumn{2}{|l|}{ Initial dose } \\
\hline $125 \mathrm{mg}$ & $71(81.6)$ \\
\hline $100 \mathrm{mg}$ & $7(8.1)$ \\
\hline 75 mg & $4(4.6)$ \\
\hline NA & $5(5.7)$ \\
\hline Median duration (range), $d$ & $259(38-1,127)$ \\
\hline \multicolumn{2}{|l|}{ Abemaciclib treatment course } \\
\hline \multicolumn{2}{|l|}{ Endocrine partner } \\
\hline Aromatase inhibitor & $24(27.6)$ \\
\hline Fulvestrant & $41(47.1)$ \\
\hline Tamoxifen & $4(4.6)$ \\
\hline Multiple & $1(1.2)$ \\
\hline NA (monotherapy) & $17(19.5)$ \\
\hline \multicolumn{2}{|l|}{ Initial dose } \\
\hline $200 \mathrm{mg}$ & $17(19.5)$ \\
\hline $150 \mathrm{mg}$ & $58(66.7)$ \\
\hline $100 \mathrm{mg}$ & $12(13.8)$ \\
\hline NA & $0(0.0)$ \\
\hline Median duration (range), $d$ & $120(2-510)$ \\
\hline
\end{tabular}

Abbreviations: CDK4/6i, cyclin-dependent kinase 4 and 6 inhibitors; NA, not available or not applicable.
Most patients received just 1 endocrine agent during their initial course of palbociclib treatment $(n=80 ; 92 \%)$ (supplemental eFigure 1A). The largest group $(n=32$; $36.8 \%$ ) transitioned from a nonsteroidal AI and palbociclib to fulvestrant and abemaciclib (supplemental eFigure 1B). In $25.3 \%$ of the cases $(n=22)$, the physicians opted to continue the same antiestrogen class (AI or fulvestrant) while changing the CDK4/6i. A total of $14.9 \%$ of patients $(n=13)$ transitioned from combination endocrine + CDK4/6i therapy to abemaciclib monotherapy.

\section{Clinical Outcomes for Patients Receiving}

Abemaciclib After Progression on a CDK4/6i

At the time of data cutoff (May 1, 2019), most patients had experienced disease progression on abemaciclib $(\mathrm{n}=58 ; 66.7 \%)$, whereas a subset remained on treatment $(n=21 ; 24.1 \%)$. A minority of patients $(n=8 ; 9.2 \%)$ discontinued abemaciclib because of toxicity without disease progression. Median PFS on abemaciclib-based therapy in the 87 patients was 5.3 months $(95 \% \mathrm{CI}$, 3.5-7.8 months; Figure 1A). Median OS on abemaciclib was 17.2 months (95\% CI, 13.2 months-not reached [NR]; Figure 1B). Thirty-two patients $(36.8 \%)$ received abemaciclib treatment for $>180$ days, including 6 who received abemaciclib monotherapy and 13 who remained on treatment at the time of analysis. Conversely, 25 patients $(28.7 \%)$ discontinued abemaciclib within 90 days of initiation because of disease progression, suggesting preexisting rather than acquired resistance to abemaciclib (including 5 patients on abemaciclib monotherapy).

No difference in median PFS was noted for patients who received abemaciclib in combination with an antiestrogen compared with those who received it as monotherapy (5.1 months [ $95 \% \mathrm{CI}, 3.2-7.6]$ vs 5.4 months [95\% CI, 1.9-NR], respectively) (supplemental eFigure 2A, B). Eight patients remained on monotherapy at the time of data cutoff, with a duration of abemaciclib treatment ranging from 2 to 460 days. To explore the relative contribution of abemaciclib to clinical benefit, patients were stratified based on the use of an antiestrogen to which they had never been exposed in the metastatic setting. Thirty patients transitioned to an antiestrogen backbone in combination with abemaciclib to which they had no prior exposure in the metastatic setting; 48 patients either received abemaciclib monotherapy or abemaciclib in combination with an antiestrogen to which they had prior exposure in the metastatic setting (and thus from which they would be expected to derive less clinical benefit). There was no meaningful difference between the PFS (5.1 vs 5.7 months) or OS (17.2 vs 15.3 months) between these groups (supplemental eFigure 3A, B).

Of note, clinical outcomes while on abemaciclib differed for patients who received the CDK4/6i sequentially 
A

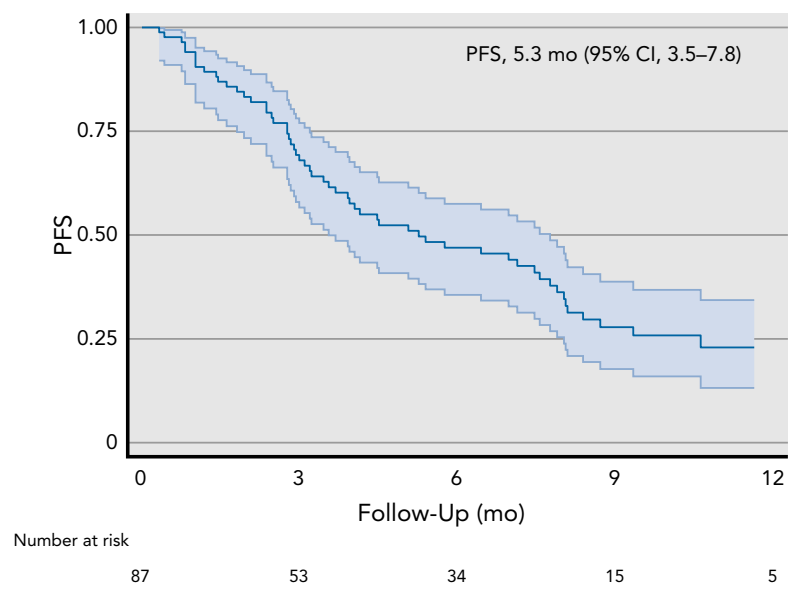

B

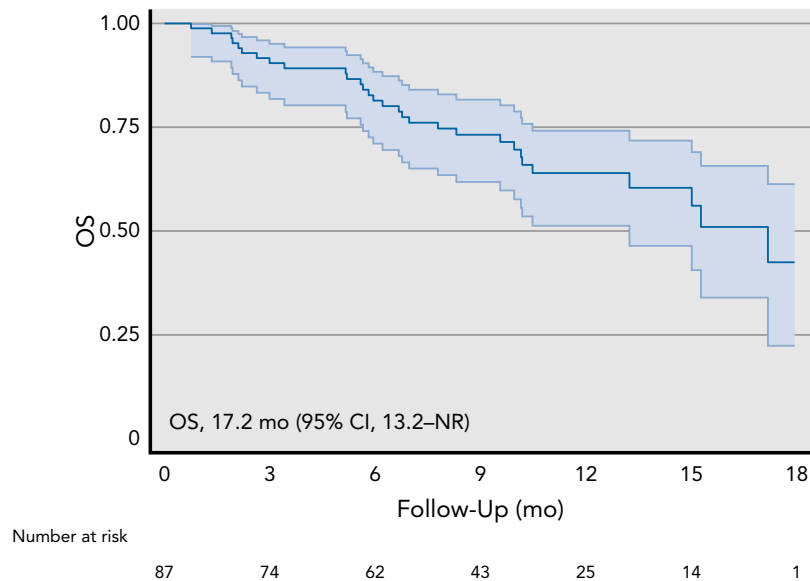

Figure 1. (A) PFS for patients receiving abemaciclib therapy after prior progression on palbociclib. (B) OS for patients receiving abemaciclib after prior progression on palbociclib therapy.

Abbreviations: NR, not reached; OS, overall survival; PFS, progression-free survival.

rather than nonsequentially. Median PFS was 8.4 months (95\% CI, 4.1-NR) in patients who received sequential CDK4/6i therapies (supplemental eFigure 4A) and 3.9 months $(95 \%$ CI, $2.9-5.7 ; P=.0013)$ in those who received nonsequential CDK4/6i therapy (supplemental eFigure 4B). This effect persisted after adjusting for the number of prior regimens the 2 groups had received, with a hazard ratio (HR) for progression of 0.42 among patients receiving sequential versus nonsequential CDK4/ $6 \mathrm{i}$ therapy $(95 \% \mathrm{CI}, 0.22-0.81$ via univariate Cox regression analysis). Furthermore, the duration of clinical treatment on prior palbociclib-based therapy did not correlate with the subsequent duration of treatment on abemaciclib-based therapy (Figure 2, supplemental eFigure 5; correlation coefficient via Spearman method, 0.065 ; $95 \% \mathrm{CI},-0.197$ to 0.318 ).

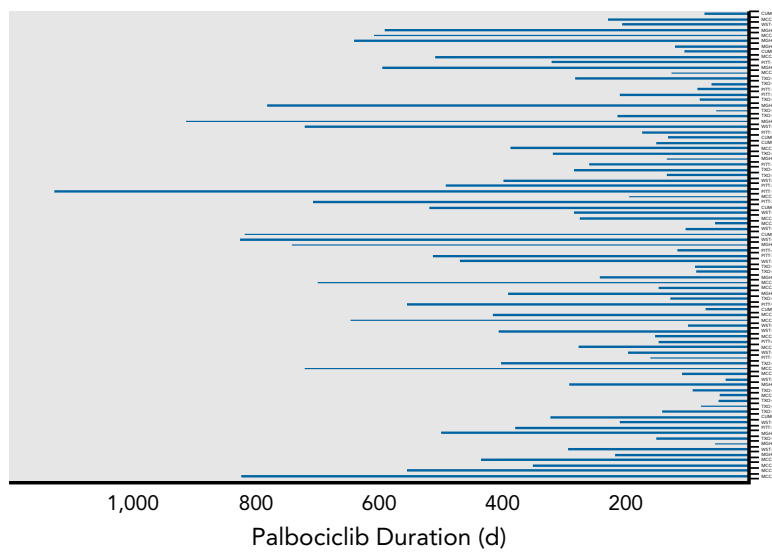

\section{Abemaciclib Tolerability After Prior CDK4/6i Exposure}

A small number of patients discontinued abemaciclib due to toxicity without disease progression $(n=8 ; 9.2 \%)$. Reasons for discontinuation included transaminitis/ hepatic injury $(n=4)$, diarrhea $(n=2)$, nausea/vomiting $(n=1)$, and weakness and tremor $(n=1)$.

Dose reductions occurred in 31 patients $(35.6 \%)$ on abemaciclib (supplemental eTable 1). Diarrhea was the most common reason for abemaciclib dose reduction $(\mathrm{n}=14 ; 16.1 \%)$, followed by fatigue $(\mathrm{n}=6 ; 6.9 \%)$ and neutropenia $(n=6 ; 6.9 \%)$.

Hematologic toxicities were common in the overall population (Table 2, supplemental eFigure 6). Anemia was the most common adverse event of interest (any grade: $72.4 \%$; grade $3-4: 14.9 \%$ ). Neutropenia (any

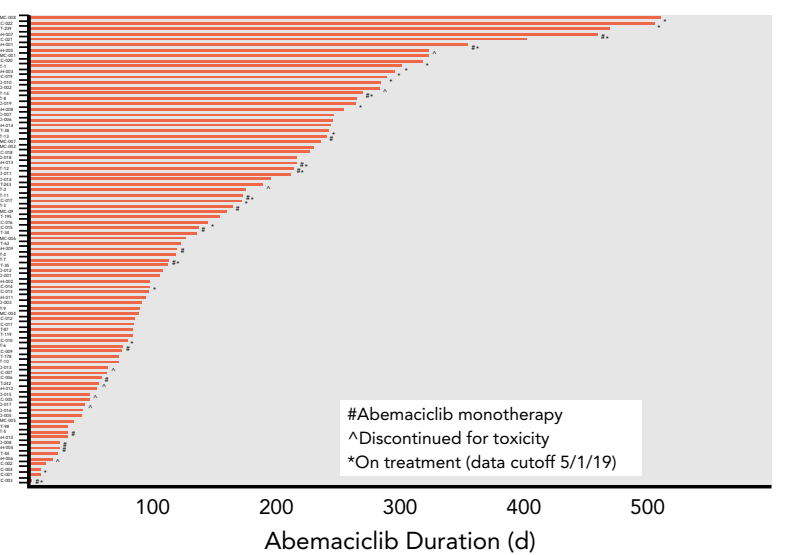

Figure 2. TTP on palbociclib-based therapy does not correlate with subsequent clinical outcome on abemaciclib. Butterfly plot demonstrating TTP on palbociclib (left) and subsequent abemaciclib (right). Patients who discontinued abemaciclib because of toxicity, received abemaciclib monotherapy, and remained on abemaciclib at data cutoff are indicated.

Abbreviation: TTP, time to progression. 
grade: $63.2 \%$; grade $3-4: 12.6 \%$ ) and thrombocytopenia (any grade: $46.0 \%$; grade 3-4: 9.2\%) were also common. Nearly one-half of the patients experienced liver function abnormalities, including elevated aspartate aminotransferase, alanine aminotransferase, and total bilirubin levels, although most of these events were grade 1 or 2 (Table 2). Increased serum creatinine levels were evident in more than one-third of patients; however, all but one event was either grade 1 or 2 . This finding is consistent with the observation that abemaciclib inhibits renal transporters (eg, OCT2, MATE1, MATE2-K) that may result in mild, reversible elevated serum creatinine levels without affecting renal function/glomerular filtration. ${ }^{29}$ Venous thromboembolism occurred in 2 patients.

\section{Identifying Resistance Mediators via}

Next-Generation Sequencing

We sought to leverage available next-generation sequencing (NGS) results to identify potential genomic predictors of early progression in patients receiving abemaciclib after prior progression on palbociclib.

Alterations in $R B 1$ were identified via targeted sequencing of circulating tumor DNA (ctDNA; Guardant assay) in 2 patients who had rapid disease progression on abemaciclib (Figure 3A, B). Targeted sequencing of ctDNA revealed additional putative resistance drivers in patients with early progression on abemaciclib, including an alteration in ERBB2 (Figure 3C) and an example of CCNE1 amplification (Figure 3D). Additional exploratory analysis of available sequencing results remains ongoing and will be presented in the future.

\section{Discussion}

This study represents the first multicenter retrospective analysis of patients with $\mathrm{HR}+\mathrm{MBC}$ and abemaciclib exposure after progression on a palbociclib-containing regimen. Abemaciclib was generally well tolerated in this
CDK4/6i-pretreated population. The overall rate of select hematologic events was similar to that reported in the phase III trials of abemaciclib in combination with endocrine therapy (MONARCH-2 and -3). ${ }^{5,6}$

Abemaciclib efficacy in these heavily pretreated patients was surprisingly similar to the results demonstrated in the MONARCH-1 trial. ${ }^{15}$ Although cross-trial comparisons need to be interpreted with caution, it is interesting to note that $36.8 \%$ of $\mathrm{CDK} 4 / 6 \mathrm{i}$-pretreated patients in this multicenter retrospective cohort continued abemaciclib for $\geq 6$ months, while the clinical benefit rate in MONARCH-1 was $42 \%$. Similarly, the median PFS was 5.3 months $(95 \%$ CI, 3.5-7.8) in the current study compared with 6.0 months in the MONARCH-1 trial (95\% CI, 4.2-7.5). Of note, although the PFS estimate in the current cohort was similar to that observed during MONARCH-1, the data presented here are limited by several factors, including sample size and the observational nature of the study. In such a retrospective analysis, the frequency of restaging studies in clinical practice can impact the estimated PFS, because scans performed less frequently outside of a clinical trial may delay the identification of progression.

Unlike MONARCH-1, in this study population, most patients received abemaciclib in combination with an antiestrogen partner. Although limited by sample size and by variations in dosing according to physician preference, there was no apparent difference in abemaciclib efficacy among patients who received abemaciclib monotherapy compared with abemaciclib in combination with hormonal therapy. Additionally, there was no clear advantage for patients who transitioned to abemaciclib in combination with a novel antiestrogen backbone compared with those who received abemaciclib monotherapy or abemaciclib in combination with an antiestrogen to which they had prior exposure in the metastatic setting. It is not likely that the endocrine therapy contributed substantially to the clinical benefit patients obtained from abemaciclib in

Table 2. AEs and Laboratory Abnormalities of Interest Associated With Abemaciclib After Prior CDK4/6i

\begin{tabular}{|c|c|c|c|c|c|c|}
\hline AE & $\begin{array}{c}\text { Grade } 1 \\
\text { n (\%) }\end{array}$ & $\begin{array}{c}\text { Grade } 2 \\
\text { n (\%) }\end{array}$ & $\begin{array}{c}\text { Grade } 3 \\
\text { n (\%) }\end{array}$ & $\begin{array}{c}\text { Grade } 4 \\
\text { n (\%) }\end{array}$ & $\begin{array}{c}\text { Any Grade } \\
\text { n (\%) }\end{array}$ & $\begin{array}{c}\text { Grade } 3 / 4 \\
\text { n (\%) }\end{array}$ \\
\hline Anemia & $27(31.0)$ & $23(26.4)$ & $13(14.9)$ & $0(0.0)$ & $63(72.4)$ & $13(14.9)$ \\
\hline Thrombocytopenia & $26(29.9)$ & $6(6.9)$ & $2(2.3)$ & $6(6.9)$ & $40(46.0)$ & $8(9.2)$ \\
\hline AST elevation & $30(34.5)$ & $6(6.9)$ & $6(6.9)$ & $0(0.0)$ & $42(48.3)$ & $6(6.9)$ \\
\hline Creatinine elevation & $25(28.7)$ & $5(5.7)$ & $1(1.2)$ & $0(0.0)$ & $31(35.6)$ & $1(1.2)$ \\
\hline VTE & $0(0.0)$ & $1(1.2)$ & $1(1.2)$ & $0(0.0)$ & $2(2.3)$ & $1(1.2)$ \\
\hline
\end{tabular}

Evaluable AEs during abemaciclib treatment were identified through medical record review. If the patient experienced multiple grades of toxicity during the abemaciclib treatment course, then the highest grade of toxicity was documented (eg, coded as grade 3 if grade 1 and 3 AEs were experienced during the course of therapy). Highest grade of toxicity experienced for each patient if multiple occurrences of the same AE.

Abbreviations: AE, adverse effect; ALT, alanine aminotransferase; AST, aspartate aminotransferase; TBIL, total bilirubin; VTE, venous thromboembolism. 


\section{A $\mathrm{MGH}_{0} 009$}

Diagnosed with T2N3

$\mathrm{ER}+/ \mathrm{PR}+/$ HER2-

breast cancer

(-)65 mo

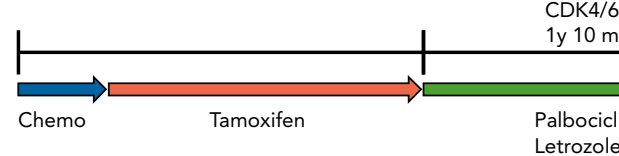

Guardant \#1 Guardant \#2

$(-) 1 \mathrm{mo} \quad(+) 1 \mathrm{mo}$

CDK $4 / 6 \quad$ CDK $4 / 6$

$\underset{\text { Palbociclib }}{\longrightarrow} \underset{\text { Abemaciclib }}{\stackrel{2 \mathrm{mo}}{\longrightarrow}}$

Palbociclib Abemaciclib Olaparib

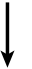

B TX0-016

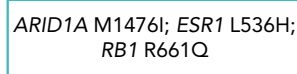

ARID1A M1476I

Diagnosed with T1N0 $\mathrm{ER}+/ \mathrm{PR}+/ \mathrm{HER} 2$

breast cancer

$(-) 142 \mathrm{mo}$

Metastatic disease

on bone biopsy

(-)61 mo

Guardant \#1

(-)0 mo

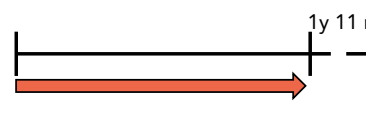

Tamoxifen
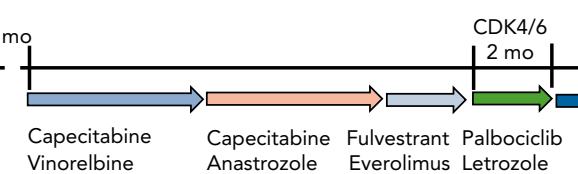

Capecitabine Fulvestrant Palbociclib Taxol $\begin{array}{llll}\text { Capecitabine } & \text { Fulvestrant Palbociclib Taxol } & \text { Abemaciclib } \\ \text { Anastrozole } & \text { Everolimus Letrozole } & \text { Carboplatin } & \text { Letrozole }\end{array}$ Vinorelbine

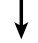

\section{PITT-5}

Diagnosed with T2N1M1 $\mathrm{ER}+/ \mathrm{PR}+/ \mathrm{HER} 2-$

metastatic breast cancer

$(-) 23$ mo

Guardant \#1

(-)9 mo

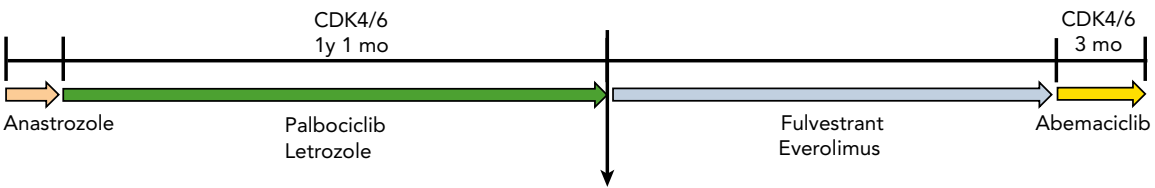

D

TXO-008

ERBB2 L869R; TP53 R273H; PIK3CA H1047R

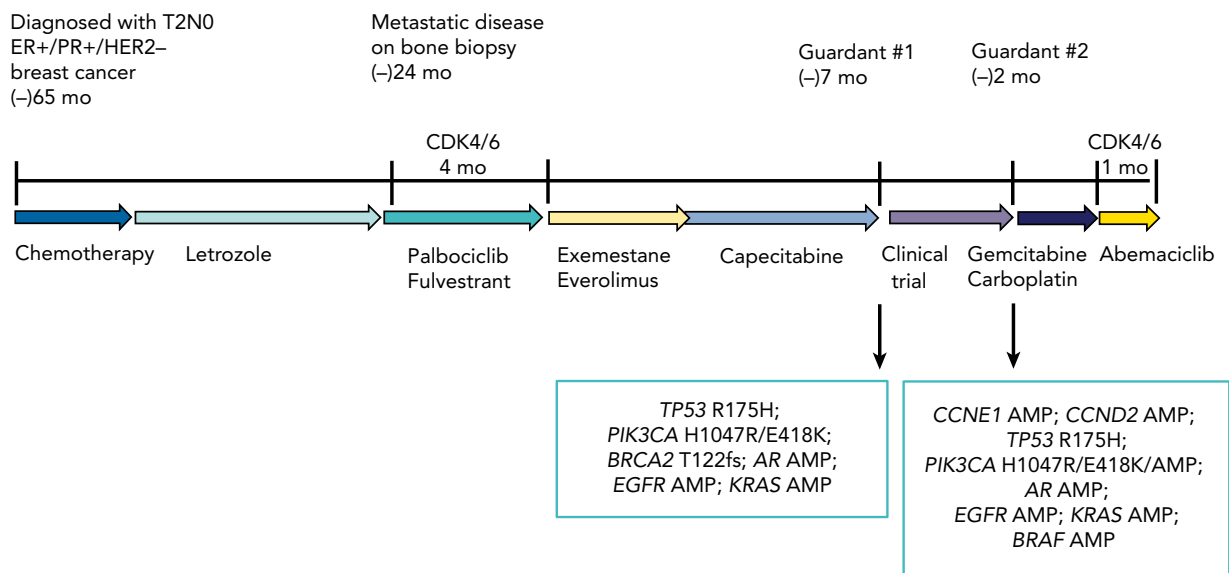

Figure 3. Clinical vignettes with next-generation sequencing provide insight into potential genomic predictors of rapid progression in patients receiving abemaciclib after prior progression on CDK4/6i. Treatment histories are provided along with results from targeted sequencing of circulating tumor DNA (Guardant assay) in representative patients. Patients who experienced rapid progression on abemaciclib harbored alterations in (A, B) RB1, (C) ERBB2, and (D) CCNE1.

Abbreviations: CDK4/6i, cyclin-dependent kinase 4 and 6 inhibitor; ER, estrogen receptor; PR, progesterone receptor. 
this study, because (1) no advantage was noted for patients who received a novel antiestrogen partner with abemaciclib, (2) many patients had prior disease progression on multiple hormonal agents, and (3) a subgroup of patients received abemaciclib monotherapy with durable clinical benefit. These findings suggest that abemaciclib may convey meaningful clinical benefit and disease control in a subset of patients who experienced progression on palbociclib.

There are important limitations to the current analysis, including the retrospective nature of the data collection, the heterogeneous patient population, and the concurrent use of abemaciclib with an antiestrogen agent for many patients. Unfortunately, many of the remaining clinical questions regarding the optimal use of CDK4/6i-based therapy in the metastatic setting are unlikely to be addressed in large, prospective, randomized trials. Although a variety of combinatorial strategies are being tested after initial progression on CDK4/6i, only 3 prospective trials are underway to address the relatively straightforward question of ongoing CDK4/6 blockade after initial progression on CDK4/6i. The phase II PALMIRA trial (ClinicalTrials.gov identifier: NCT03809988) is exploring the utility of palbociclib continuation with a transition in the endocrine backbone for patients who derived clinical benefit from a prior course of palbociclibbased treatment. The phase II PACE trial (NCT03147287) is designed to compare fulvestrant monotherapy with fulvestrant + palbociclib following initial progression on a CDK4/6i with an AI; a third arm on the PACE trial is exploring the utility of fulvestrant and palbociclib combined with the PD-1 inhibitor avelumab. Lastly, the phase II MAINTAIN study (NCT02632045) similarly explores the utility of ribociclib with fulvestrant following progression on an initial CDK4/6i with an AI. Neither PALMIRA, PACE, nor MAINTAIN is evaluating the potential utility of abemaciclib in a CDK4/6i-pretreated patient population. The ongoing phase Ib JBPH trial (NCT02057133) includes an arm for women with $\mathrm{HR}+\mathrm{MBC}$ whose disease had progressed on a CDK4/6i and an antiestrogen agent; the same antiestrogen is being continued while the patient transitions to abemaciclib. These results, when available, will provide insight into the utility of abemaciclib plus continued estrogen receptor inhibition after CDK4/6i progression, but will not address the efficacy of abemaciclib monotherapy nor combination therapy with an alternate antiestrogen, the 2 options that were preferred within the context of our retrospective analysis. Hence, despite these 4 studies, it is unlikely that this question-the utility of abemaciclib following progression on palbociclib or ribociclib - will be adequately answered in the context of an ongoing prospective, randomized study.

Given the widespread use of CDK4/6i agents in the large $\mathrm{HR}+\mathrm{MBC}$ population, it will be critical to identify biomarkers that help identify patients who may benefit from a second course of CDK4/6i-based therapy. Early preclinical efforts (both in vitro and in xenografts) have suggested that $\mathrm{HR}+$ breast cancer cells that develop acquired resistance to palbociclib are also cross-resistant to abemaciclib in the laboratory. ${ }^{28,30}$ Additional translational work to date has shed light on the heterogeneous landscape of resistance to CDK4/6i inhibitors. Acquired disruption of $R B 1$ function has been demonstrated in a small number of patients at the time of resistance. ${ }^{22-25}$ Regulation of CDK6, via the Hippo pathway or microRNAdependent signaling, has also been implicated in the development of resistance. ${ }^{24,26} C C N E 1$ overexpression was a predictor of inferior response to palbociclib, whereas loss of the PTEN tumor suppressor was demonstrated after progression on ribociclib-based therapy. ${ }^{27,31}$ The FGFR, ERBB2, AKT1, aurora kinase, and RAS pathways have also been implicated as potential CDK4/6i resistance mediators both preclinically and in patient samples. ${ }^{20,21,28,32}$

\section{Conclusions}

This multicenter, retrospective analysis represents the first effort to investigate the potential for continued CDK4/6 blockade, via abemaciclib treatment, following disease progression on palbociclib in patients with $\mathrm{HR}+\mathrm{MBC}$. Abemaciclib use after prior CDK4/6i treatment was well tolerated and, in this heavily pretreated population, clinical benefit (with at least a 6-month treatment duration) was identified in a clinically meaningful subset of patients (36.8\%). Overall PFS and OS in this cohort were similar to those observed in the MONARCH-1 study, in which none of the patients had received prior CDK4/6i therapy. Furthermore, ctDNA sequencing allowed identification of multiple genomic effectors, previously implicated in CDK4/6i resistance, in patients who had rapid disease progression on abemaciclib. Overall, these findings suggest that some patients may benefit from continued CDK4/6-directed therapy and support the need for additional blood- and tissue-based studies to guide therapeutic selection for this important patient population.

Submitted May 18, 2020; accepted for publication September 28, 2020.

Author contributions: Study concept and design: Wander, Han, Brufsky, Kalinsky, Ma, O'Shaughnessy, Bardia. Data acquisition and provision of study materials: All authors. Initial manuscript draft: Wander, Han, Zangardi, Niemierko, Brufsky, Kalinsky, Ma, O'Shaughnessy, Bardia. Critical feedback and development of final manuscript: All authors.

Disclosures: Dr. Wander has reported serving as a consultant for Foundation Medicine, Veracyte, Eli Lilly, and Puma Biotechnology. Dr. Han has reported receiving grant/research support from Arvinas, AbbVie, Bristol-Myers Squibb, Daiichi Pharma, G1 therapeutics, GlaxoSmithKline, Horizon, Karyopharm, Marker Therapeutics, Novartis, Pfizer, Prescient, Seattle Genetics, and Zymeworks, and serving on the speakers' bureau of Eli Lilly. Dr. Kambadakone has reported receiving grant/research support from Philips Healthcare and GE Healthcare. Dr. Moy has reported receiving grant/research support from Puma Biotechnology. Dr. Spring has reported serving as a consultant/advisory board member for Novartis, Lumicell, Puma Biotechnology; receiving travel fees from Merck and Tesaro; and receiving grant/research support from Merck and Tesaro. Dr. Vidula has reported receiving grant/research support from Radius, Daehwa, Merck, Pfizer, and Novartis, and serving on the advisory board of AbbVie. Dr. Wagle has reported serving as a consultant and being a 
former stockholder of Foundation Medicine; serving as a consultant and receiving grant/research support from Novartis; receiving research support from PUMA biotechnology; serving on the advisory board for Section 32; serving on the advisory board and being a stockholder for Relay Therapeutics; and serving as a consultant for Eli Lilly. Dr. Brufsy has reported serving as a scientific advisor for Pfizer and Eli Lilly. Dr. Kalinsky has reported having a spouse who is an employee at Pfizer; holding stock in Array BioPharma; serving as an advisor/consultant for Biotheranostics, Eli Lilly, Pfizer, Novartis, Eisai, AstraZeneca, Merck, Seattle Genetics, and Cyclacel; serving on the speakers' bureau for Eli Lilly; and receiving grant/research support from Incyte, Genentech, Eli Lilly, Pfizer, Calithera Biosciences, Acetylon, Seattle Genetics, Amgen, Zeno Pharmaceuticals, and CytomX Therapeutics. Dr. Ma has reported receiving grant/research support from Pfizer and Puma Biotechnology, and receiving consulting fees from AstraZeneca, Seattle Genetics, Agendia, Eli Lilly, Novartis, Tempus, and Pfizer. Dr. O'Shaughnessy has reported receiving honoraria and consulting fees from AbbVie Inc., Agendia, AstraZeneca, Bristol-Myers
Squibb, Celgene Corporation, Eisai, Genentech, Genomic Health, GRAIL, Immunomedics, Heron Therapeutics, Ipsen Biopharmaceuticals, Jounce Therapeutics, Eli Lilly, Merck, Myriad, Novartis, Ondonate Therapeutics, Pfizer, Puma Biotechnology, Prime Oncology, Roche, Seattle Genetics, and Syndax Pharmaceuticals. Dr. Bardia has disclosed serving as a consultant/advisory board member for Pfizer, Novartis, Genentech, Merck, Radius Health, Immunomedics, Taiho, Sanofi, Diiachi Pharma/AstraZeneca, Puma, Biothernostics Inc., Phillips, Eli Lilly, and Foundation Medicine, and receiving grant/research support from Genentech, Novartis, Pfizer, Merck, Sanofi, Radius Health, Immunomedics, and Diiachi Pharma/Astra Zeneca. The remaining authors have disclosed that they have not received any financial consideration from any person or organization to support the preparation, analysis, results, or discussion of this article.

Correspondence: Aditya Bardia, MD, MPH, Massachusetts Genera Hospital Cancer Center, 55 Fruit Street, Boston, MA 02114-2696. Email: bardia.aditya@mgh.harvard.edu

\section{References}

1. Spring LM, Wander SA, Zangardi M, et al. CDK 4/6 inhibitors in breast cancer: current controversies and future directions. Curr Oncol Rep 2019, 21:25

2. Finn RS, Martin M, Rugo HS, et al. Palbociclib and letrozole in advanced breast cancer. N Engl J Med 2016;375:1925-1936.

3. Cristofanilli M, Turner NC, Bondarenko I, et al. Fulvestrant plus palbociclib versus fulvestrant plus placebo for treatment of hormone-receptor-positive, HER2-negative metastatic breast cancer that progressed on previous endocrine therapy (PALOMA-3): final analysis of the multicentre, double-blind, phase 3 randomised controlled trial. Lancet Oncol 2016;17:425-439.

4. Hortobagyi GN, Stemmer SM, Burris HA, et al. Ribociclib as first-line therapy for HR-positive, advanced breast cancer. N Engl J Med 2016;375:1738-1748.

5. Goetz MP, Toi M, Campone M, et al. MONARCH 3: abemaciclib as initial therapy for advanced breast cancer. J Clin Oncol 2017;35:3638-3646.

6. Sledge GW Jr, Toi M, Neven $P$, et al. MONARCH 2: abemaciclib in combination with fulvestrant in women with $\mathrm{HR}+/ \mathrm{HER} 2-$ advanced breast cancer who had progressed while receiving endocrine therapy. J Clin Oncol 2017;35:2875-2884

7. Sledge GW Jr, Toi M, Neven P, et al. The effect of abemaciclib plus fulvestrant on overall survival in hormone receptor-positive, erbb2negative breast cancer that progressed on endocrine therapy-MONARCH 2: a randomized clinical trial. JAMA Oncol 2019;6:116-124.

8. Im SA, Lu YS, Bardia A, et al. Overall survival with ribociclib plus endocrine therapy in breast cancer. N Engl J Med 2019;381:307-316.

9. Slamon D, Neven P, Chia S, et al. Overall survival results of the phase III MONALEESA-3 trial of postmenopausal patients with hormone receptorpositive, human epidermal growth factor 2-negative advanced breast cancer treated with fulvestrant \pm ribociclib. Ann Oncol 2019;30(Suppl 5):v851-934.

10. Turner NC, Slamon DJ, Ro J, et al. Overall survival with palbociclib and fulvestrant in advanced breast cancer. N Engl J Med 2018;379:1926-1936.

11. Finn RS, Crown J, Lang I, et al. Overall survival results from the randomized phase II study of palbociclib $(P)$ in combination with letrozole $(L)$ vs letrozole alone for frontline treatment of ER+/HER2- advanced breast cancer (PALOMA-1: TRIO-18). Breast Cancer Res Treat 2020:183:419-428.

12. Sanchez-Martinez C, Gelbert LM, Shannon H, et al. LY2835219, a potent oral inhibitor of the cyclin-dependent kinases 4 and 6 (CDK4/6) that crosses the blood-brain barrier and demonstrates in vivo activity against intracranial human brain tumor xenografts [abstract]. Mol Cancer Ther 2011;10:Abstract B234.

13. Tolaney SM, Lin NU, Thornton $D$, et al. Abemaciclib for the treatment of brain metastases (BM) secondary to hormone receptor positive $(\mathrm{HR}+)$, HER2 negative breast cancer [abstract]. J Clin Oncol 2017;35(Suppl):Abstract 1019.

14. Hafner M, Mills CE, Subramanian K, et al. Multiomics profiling establishes the polypharmacology of FDA-approved CDK4/6 inhibitors and the potential for differential clinical activity. Cell Chem Biol 2019;26:1067-1080.

15. Dickler MN, Tolaney SM, Rugo HS, et al. MONARCH 1, a phase II study of abemaciclib, a CDK4 and CDK6 inhibitor, as a single agent, in patients with refractory HR+/HER2- metastatic breast cancer. Clin Cancer Res 2017;23:5218-5224.

16. Herrera-Abreu MT, Palafox M, Asghar U, et al. Early adaptation and acquired resistance to CDK4/6 inhibition in estrogen receptor-positive breast cancer. Cancer Res 2016;76:2301-2313.
17. Yang C, Li Z, Bhatt T, et al. Acquired CDK6 amplification promotes breast cancer resistance to CDK4/6 inhibitors and loss of ER signaling and dependence. Oncogene 2017;36:2255-2264.

18. Caldon CE, Sergio CM, Kang J, et al. Cyclin E2 overexpression is associated with endocrine resistance but not insensitivity to CDK2 inhibition in human breast cancer cells. Mol Cancer Ther 2012;11:1488-1499.

19. Formisano L, Lu Y, Servetto A, et al. Aberrant FGFR signaling mediates resistance to CDK4/6 inhibitors in ER+ breast cancer. Nat Commun 2019 10:1373.

20. Drago JZ, Formisano L, Juric D, et al. FGFR1 gene amplification mediates endocrine resistance but retains TORC sensitivity in metastatic hormone receptor positive (HR+) breast cancer. Clin Cancer Res 2019;25:6443-6451.

21. Nayar U, Cohen $O$, Kapstad $C$, et al. Acquired HER2 mutations in ER+ metastatic breast cancer confer resistance to estrogen receptor-directed therapies. Nat Genet 2019;51:207-216.

22. Condorelli R, Spring L, O'Shaughnessy J, et al. Polyclonal RB1 mutations and acquired resistance to CDK $4 / 6$ inhibitors in patients with metastatic breast cancer. Ann Oncol 2018:29:640-645.

23. O'Leary B, Cutts RJ, Liu Y, et al. The genetic landscape and clonal evolution of breast cancer resistance to palbociclib plus fulvestrant in the PALOMA-3 trial. Cancer Discov 2018;8:1390-1403.

24. Li Z, Razavi P, Li Q, et al. Loss of the FAT1 tumor suppressor promotes resistance to CDK4/6 inhibitors via the Hippo pathway. Cancer Cell 2018; 34:893-905.

25. Wander S, Cohen O, Johnson G, et al. Whole exome sequencing (WES) in hormone-receptor positive (HR+) metastatic breast cancer (MBC) to identify mediators of resistance to cyclin-dependent kinase $4 / 6$ inhibitors (CDK4/6i) [abstract]. J Clin Oncol 2018;36(Suppl):Abstract 12016.

26. Cornell L, Wander SA, Visal T, et al. MicroRNA-mediated suppression of the TGF-B pathway confers transmissible and reversible CDK4/6 inhibitor resistance. Cell Rep 2019;26:2667-2680.

27. Turner NC, Liu Y, Zhu Z, et al. Cyclin E1 expression and palbociclib efficacy in previously treated hormone receptor-positive metastatic breast cancer $\mathrm{J}$ Clin Oncol 2019;37:1169-1178.

28. Wander SA, Cohen O, Gong X, et al. The genomic landscape of intrinsic and acquired resistance to cyclin-dependent kinase 4/6 inhibitors in patients with hormone receptor positive metastatic breast cancer. Cancer Discov 2020;10:1174-1193.

29. Chappell JC, Turner PK, Pak YA, et al. Abemaciclib inhibits renal tubular secretion without changing glomerular filtration rate. Clin Pharmacol Ther 2019;105:1187-1195.

30. O'Brien NA, McDermott MSJ, Conklin D, et al. Targeting activated PI3K/ mTOR signaling overcomes acquired resistance to CDK4/6-based therapies in preclinical models of hormone receptor-positive breast cancer. Breast Cancer Res 2020;22:89.

31. Costa $C$, Wang $Y$, Ly A, et al. PTEN loss mediates clinical cross-resistance to CDK4/6 and PI3K $\alpha$ inhibitors in breast cancer. Cancer Discov 2020;10:72-85.

32. Mao P, Cohen O, Kowalski KJ, et al. Acquired FGFR and FGF alterations confer resistance to estrogen receptor (ER) targeted therapy in ER+ metastatic breast cancer. Clin Cancer Res 2020;26:5974-5989. 
Supplemental online content for:

\section{Clinical Outcomes With CDK4/6 Inhibitor Abemaciclib After Prior CDK4/6 Inhibitor Use in Breast Cancer: A Multicenter Experience}

Seth A. Wander, MD, PhD; Hyo S. Han, MD; Mark L. Zangardi, PharmD; Andrzej Niemierko, PhD; Veronica Mariotti, MD; Leslie S.L. Kim, BS; Jing Xi, MD; Apurva Pandey, MD; Siobhan Dunne, BA; Azadeh Nasrazadani, MD, PhD; Avinash Kambadakone, MD; Casey Stein, BS; Maxwell R. Lloyd, BS; Megan Yuen, BS; Laura M. Spring, MD; Dejan Juric, MD; Irene Kuter, MD; loannis Sanidas, PhD; Beverly Moy, MD, MPH; Therese Mulvey, MD; Neelima Vidula, MD; Nicholas J. Dyson, PhD; Leif W. Ellisen, MD, PhD; Steven Isakoff, MD, PhD; Nikhil Wagle, MD, PhD; Adam Brufsky, MD, PhD; Kevin Kalinsky, MD; Cynthia X. Ma, MD, PhD; Joyce O'Shaughnessy, MD; and Aditya Bardia, MD, MPH

J Natl Compr Canc Netw, doi: 10.6004/jnccn.2020.7662

eFigure 1: Proportion of Patients Receiving Antiestrogen

eFigure 2: Clinical Outcomes on Abemaciclib in Patients Receiving Monotherapy Versus Hormone Therapy eFigure 3: Clinical Outcomes on Abemaciclib After Change Versus No Change in Antiestrogen Backbone eFigure 4: Median PFS After Stratification Based on Whether Patients Received CDK4/6i Therapy Sequentially or Nonsequentially

eFigure 5: Correlation Between Time to Progression on Palbociclib-Based Therapy and on Abemaciclib eFigure 6: Incidence of Adverse Effects of Interest During Abemaciclib Treatment

eTable 1: Abemaciclib Dose Reductions

eAppendix 1: Supplemental Methods 
A

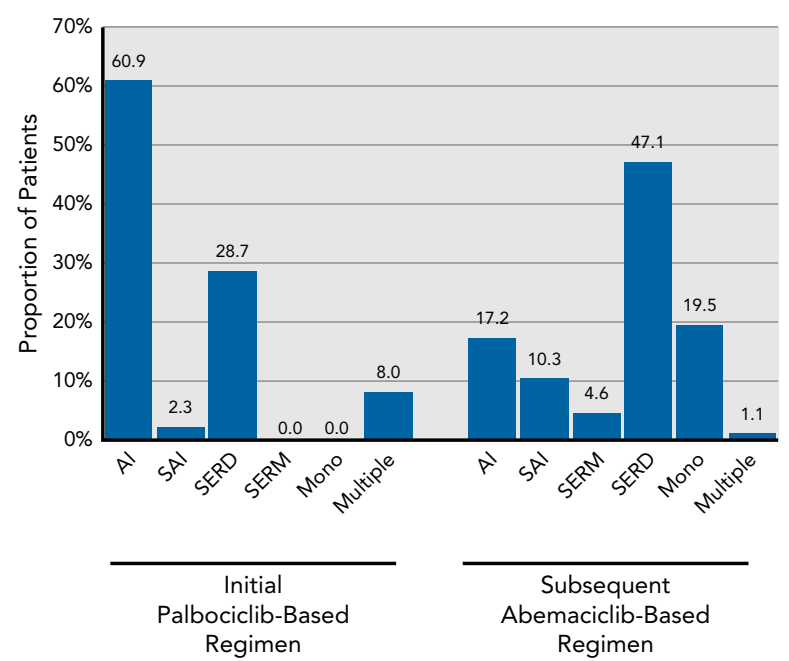

B

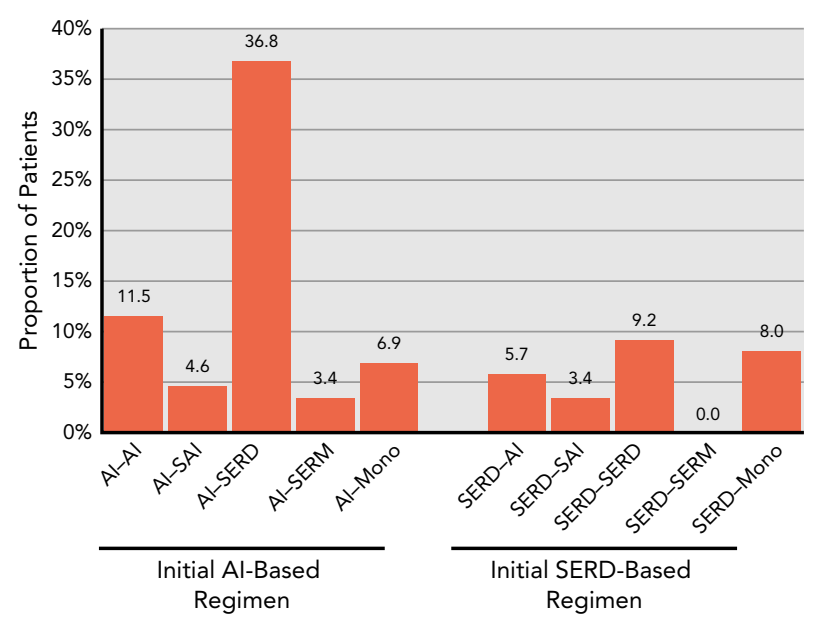

eFigure 1. (A) Proportion of patients receiving the indicated antiestrogen in the initial palbociclib-based regimen and subsequent abemaciclibbased regimen. (B) Proportion of patients with each pattern of antiestrogen transition (between the 2 CDK4/6i regimens) for treatment with palbociclib with an Al or with fulvestrant.

Abbreviations: Al, (nonsteroidal) aromatase inhibitor; CDK4/6i, cyclin-dependent kinase 4 and 6 inhibitor; mono, monotherapy; SAl, steroidal aromatase inhibitor SERD, selective estrogen receptor degrader (fulvestrant); SERM, selective estrogen receptor modulator (tamoxifen).

A

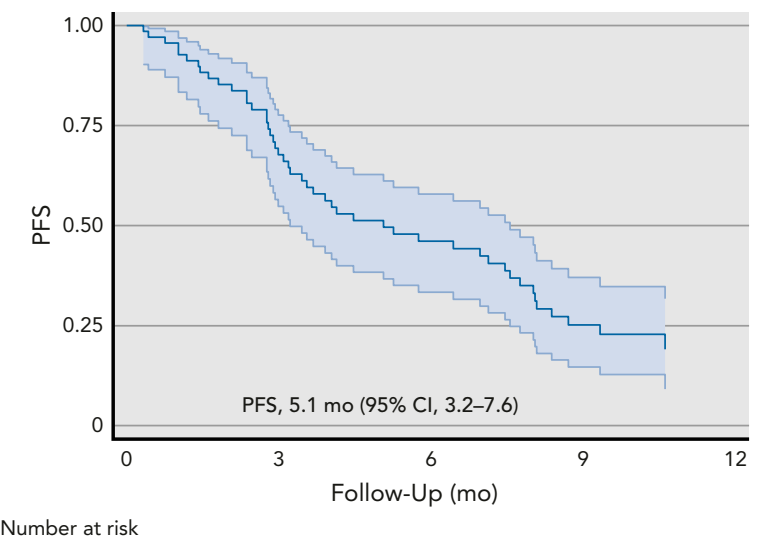

B

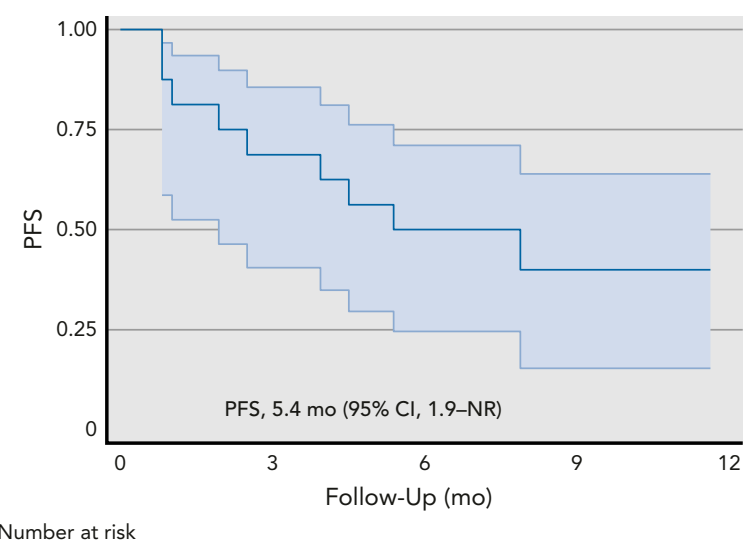

Number at risk

3

eFigure 2. Clinical outcomes on abemaciclib in patients receiving monotherapy versus hormone therapy. PFS results after stratification based on whether patients received abemaciclib therapy $(A)$ in combination with an antiestrogen or $(B)$ as monotherapy did not demonstrate any meaningful difference.

Abbreviations: mono, monotherapy; NR, not reached; PFS, progression-free survival. 
A

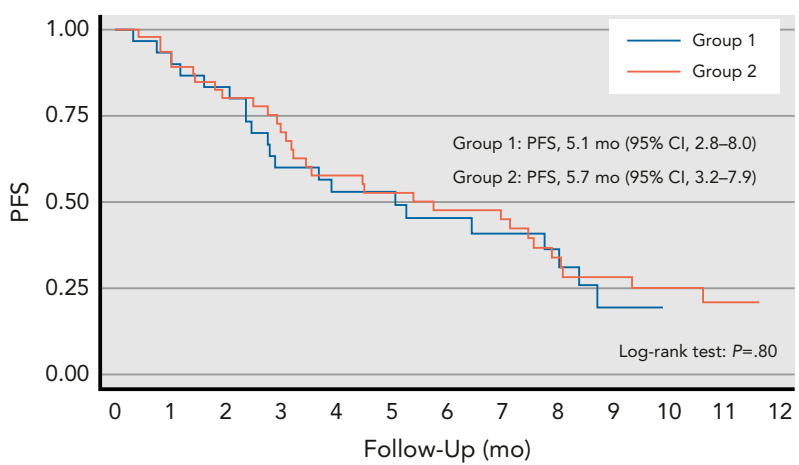

Number at risk

$\begin{array}{llllllllllllll}\text { Group } 1 & 30 & 28 & 25 & 18 & 15 & 14 & 11 & 9 & 7 & 3 & 1 & 1 & 1\end{array}$
B

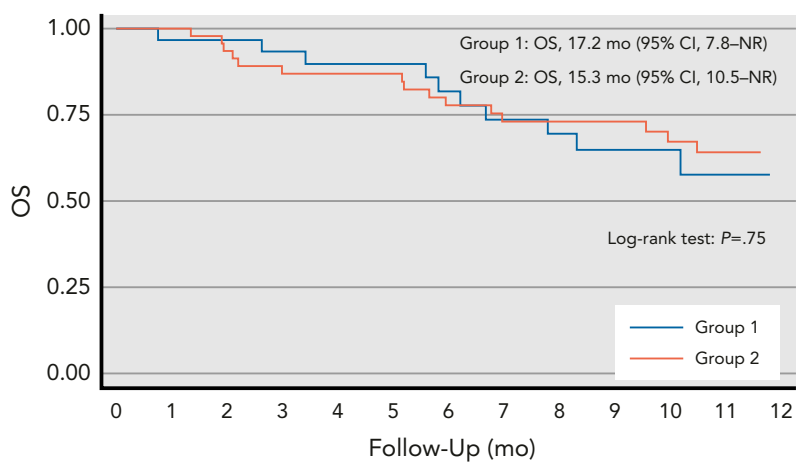

Number at risk

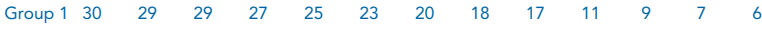

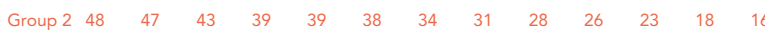

eFigure 3. Clinical outcomes on abemaciclib after change versus no change in antiestrogen backbone. (A) PFS and (B) OS in patients for whom a change in the class of antiestrogen backbone was made when switching from palbociclib to abemaciclib (Group 1$)$ and those who received abemaciclib monotherapy or abemaciclib in combination with an antiestrogen to which they had previously been exposed in the metastatic setting (Group 2).

Abbreviations: NR, not reached; OS, overall survival; PFS, progression-free survival.

A

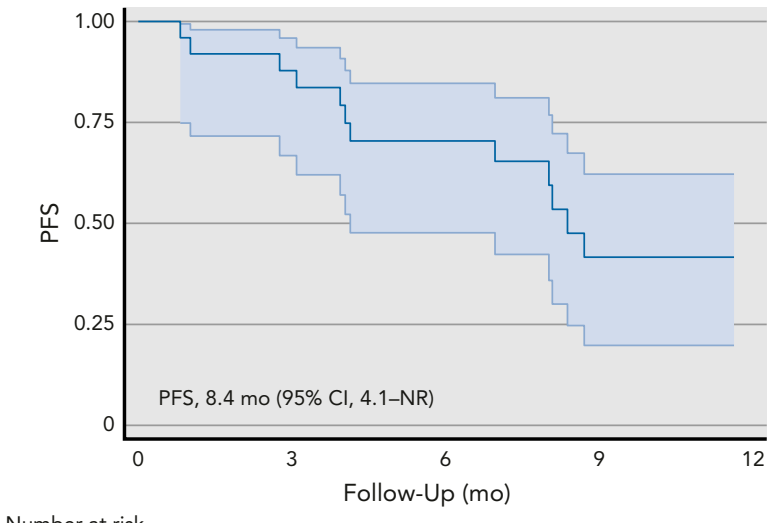

Number at risk

25
21
B

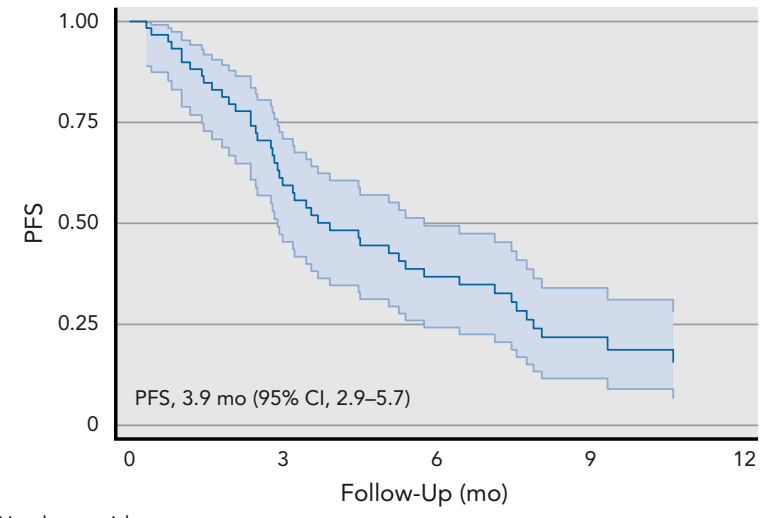

Number at risk

32

19 8 4

eFigure 4. Median PFS after stratification based on whether patients received CDK4/6i therapy (A) sequentially or (B) nonsequentially suggested that patients receiving sequential therapy derived greater clinical benefit.

Abbreviations: CDK4/6i, cyclin-dependent kinase 4 and 6 inhibitor; NR, not reached; PFS, progression-free survival. 


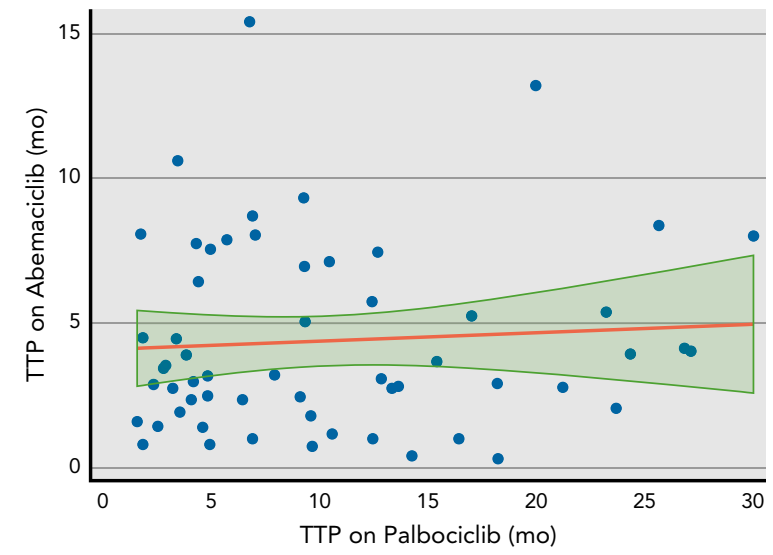

eFigure 5. TTP on palbociclib-based therapy does not correlate with subsequent clinical outcome on abemaciclib. Abbreviation: TTP, time to progression.

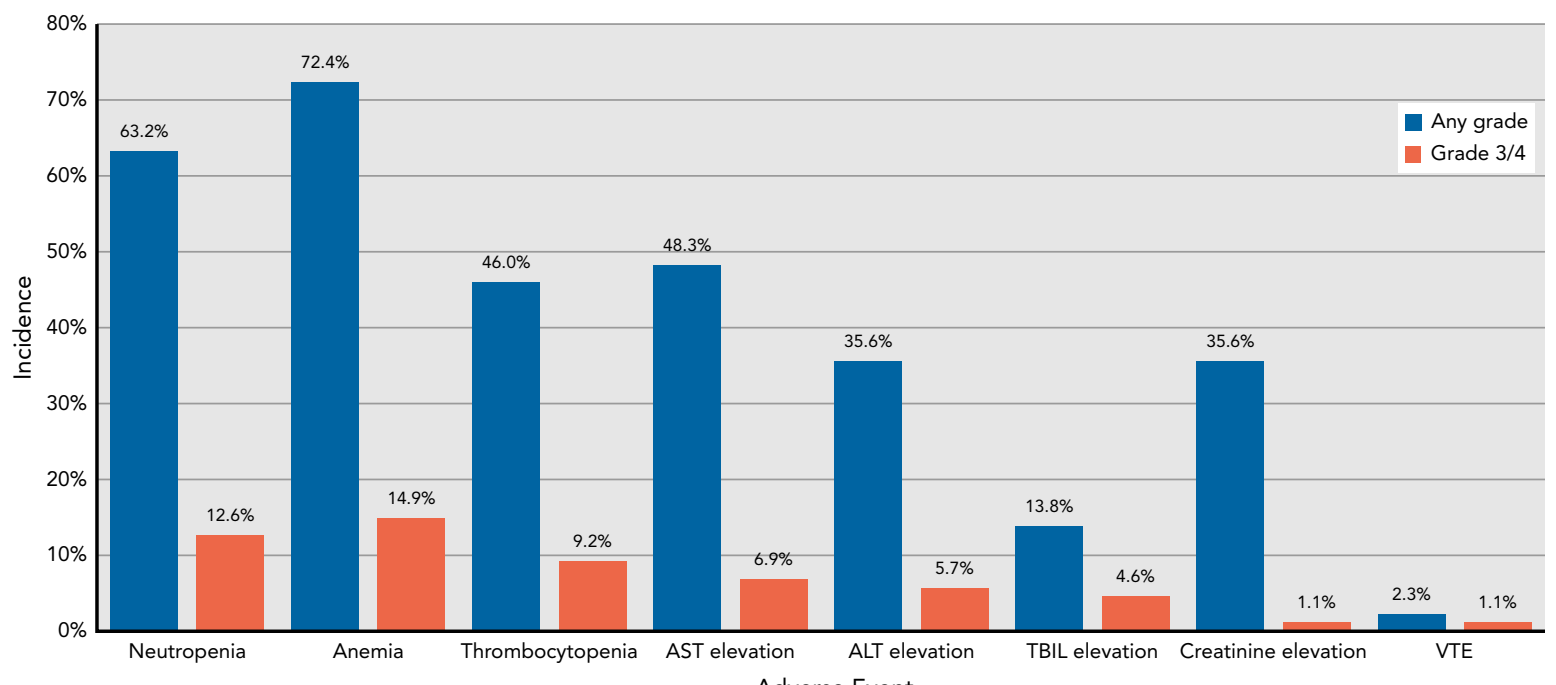

$$
\text { Adverse Event }
$$

eFigure 6. Incidence of AEs of interest during abemaciclib treatment.

Abbreviations: AE, adverse effect; ALT, alanine aminotransferase; AST, aspartate aminotransferase; TBIL, total bilirubin; VTE, venous thromboembolism. 


\section{4 - Wander et al}

\section{eTable 1. Abemaciclib Dose Reductions}

$\mathbf{n}$

\begin{tabular}{|lc|}
\hline Single dose reduction & $31(35.6 \%)$ \\
\hline Two dose reductions & $5(5.8 \%)$ \\
\hline Indication for dose reduction, per EMR review ${ }^{\mathrm{a}}$ & 14 \\
\hline Diarrhea & 6 \\
\hline Neutropenia & 6 \\
\hline Fatigue & 5 \\
\hline Nausea/Vomiting & 2 \\
\hline Thrombocytopenia & 2 \\
\hline Transaminitis & 2 \\
\hline NA & 1 \\
\hline Cough & 1 \\
\hline Headache & 1 \\
\hline Weakness & 2 \\
\hline
\end{tabular}

Abbreviations: EMR, electronic medical record; NA, not available.

aFor some patients, multiple indications for dose reductions were cited. 


\section{eAppendix 1. Supplemental Methods}

Given that the primary objective was to evaluate the role of abemaciclib-based therapy in patients whose disease had progressed on prior inhibitors of cyclin-dependent kinases 4 and 6 (CDK4/6i; palbociclib or ribociclib) and to maintain homogeneity in the eligible population, we included patients who experienced progression while on the initial course of CDK4/6i but not those who discontinued the initial CDK4/6i because of toxicity or who had received abemaciclib as the initial CDK4/6i. Similarly, patients who received $>1$ line of CDK4/6i regimen prior to receiving abemaciclib or who received CDK4/6i in the context of a triplet regimen on a clinical trial (such as endocrine therapy with CDK4/6i and an mTOR inhibitor) were also excluded.

Patients were included if they received abemaciclib sequentially (immediately after disease progression on the initial CDK4/6i) or nonsequentially (after at least 1 intervening systemic therapy after progression on the initial CDK4/6i). Abemaciclib could have been administered in combination with an antiestrogen agent or as monotherapy. Patients with HER2-positive or triple-negative disease were excluded.

Dose, schedule, and dose reduction information for both CDK4/6i regimens were captured, along with treatment duration, whether the CDK4/6i regimens were given sequentially or nonsequentially, the number of metastatic therapy regimens given prior to the initiation of abemaciclib, the current status of the abemaciclib-based regimen (on or off treatment), reason for discontinuation (disease progression or toxicity, per medical record and radiology review), date of last follow-up, and survival status.

Medical records (progress notes, laboratory values) for each patient were reviewed to identify select adverse events and laboratory abnormalities associated with abemaciclib. 